\title{
Das öffentlich geförderte Theater in der Legitimationskrise und unter Innovationsdruck? Ergebnisse einer repräsentativen Bevölkerungsbefragung
}

\section{Birgit Mandel}

\section{Zusammenfassung}

Die Stadt- und Staatstheater als die am höchsten geförderten Kultureinrichtungen in Deutschland stehen in besonderer Weise unter Legitimationsdruck und benötigen ausreichend Rückhalt bei relevanten Stakeholdern, bei Publikum und in der Bevölkerung. Welches Interesse an Theatern und welche Einstellungen zu deren Aufgaben und deren öffentlicher Förderung gibt es in der Bevölkerung in Deutschland? Der Artikel präsentiert und diskutiert die Ergebnisse einer repräsentativen Bevölkerungsbefragung, die zeigt, dass zwar nur wenige zu den regelmäßigen Besucher*innen von Theatern gehören - darunter eher hoch gebildete, eher ältere und eher weibliche Personen - eine große Bevölkerungsmehrheit sich jedoch für eine weiterhin hohe öffentliche Förderung ausspricht. Von den Theatern werden vor allem eine hohe Zugänglichkeit sowie Programme kultureller Bildung für Kinder und Jugendliche erwartet. Die hohe Zustimmung zur Theaterförderung, weit über den Kreis des Publikums hinaus, verweist auf die hohe gesellschaftliche Bedeutung, die den Theatern zugeschrieben wird. Zugleich deuten sich erste Risse dieses positiven Theaterbildes bei den jüngeren Generationen an.

B. Mandel (凶)

Institut für Kulturpolitik, Universität Hildesheim, Hildesheim, Deutschland E-Mail: mandel@uni-hildesheim.de 


\section{Schlüsselwörter}

Bevölkerungsbefragung • Theaterförderung $\bullet$ Legitimation $•$ Kulturelle Teilhabe

\section{$1 \quad$ Einleitung}

Die Stadt- und Staatstheater, in Deutschland traditionell Mittelpunkt des öffentlichen Kulturlebens in größeren Städten, benötigen die Legitimierung durch die „Stadtgesellschaft", um ihre herausgehobene Position in der öffentlichen Kultur-Förderpolitik zu behaupten. Angesichts einer stark veränderten Bevölkerung mit diversifizierten kulturellen Präferenzen und Rezeptionsweisen scheint eine Zustimmung zur Förderung der Theater keineswegs mehr selbstverständlich. Befinden sich Stadt- und Staatstheater also in einer Legitimationskrise, weil das Interesse an und die Nachfrage nach Theaterangeboten sowie die Einschätzung der Theater als persönlich bereichernde oder zumindest als gesellschaftlich wertvolle Einrichtungen in der Bevölkerung möglicherweise nicht (mehr) hinreichend vorhanden sind? Und was macht den gesellschaftlichen Wert der Stadtund Staatstheater aus? Liegt dieser im Nachweis künstlerischer Exzellenz oder künstlerischer Innovation, geht es um die Pflege klassischer Werke, oder um die Befriedigung des Wunsches nach guter Unterhaltung? Soll öffentlich gefördertes Theater Angebote kultureller Bildung bereitstellen oder mehr noch sich um kulturelle Inklusion und Gemeinschaftsstiftung in einer Stadt bemühen? Die Bandbreite unterschiedlicher und zum Teil auch widersprüchlicher Erwartungen an die öffentlichen Theater scheint groß.

Kulturpolitik in Deutschland orientierte sich seit den 1950er Jahren in ihrer Förderpolitik im Wesentlichen an den klassischen Kunstinstitutionen und hier vor allem am Theater. Klassische Kultur und zeitgenössische Kunstproduktion wurden in Deutschland in öffentlichen Einrichtungen mit einer weltweit einzigartigen kulturellen Infrastruktur fest etabliert - weitgehend unter Ausschluss von Marktmechanismen. Zentrales Begründungsmuster für Kulturpolitik in Deutschland war und ist die grundgesetzlich verankerte Kunstfreiheitsgarantie. Implizit daraus abgeleitet wird der Anspruch diese zu ermöglichen durch eine hohe öffentliche Förderung und Bereitstellung einer breiten öffentlichen Infrastruktur für Kunst und Kultur bei gleichzeitig hoher Zurückhaltung in der politischen Steuerung der öffentlich finanzierten Einrichtungen. Seit den 1970er Jahren etablierte sich zudem das Paradigma einer teilhabeorientierten „Kulturpolitik als Gesellschaftspolitik“ der sogenannten „Neuen Kulturpolitik“, die über die Förderung 
der „schönen Künste“ hinaus einen zentralen Beitrag zur Demokratisierung der Gesellschaft leisten müsse. Eng damit verbunden ist das Entstehen neuer sozialer Bewegungen und der Soziokultur mit ihrem breiten, auch alltagskulturell verankerten Kulturbegriff jenseits der klassischen Kultureinrichtungen. Auch wenn sich infolgedessen in Deutschland sehr viele soziokulturelle Einrichtungen etablierten, änderten sie wenig an der weit überdimensionalen Förderung der traditionellen Kultureinrichtungen durch staatliche Kulturpolitik.

Übergreifende Trends wie Globalisierung, Migration, Digitalisierung sowie eine Tendenz zur Individualisierung der Gesellschaft haben Auswirkungen auf kulturelle Interessen, Rezeptionsweisen von Kunst und Kultur und kulturelle Partizipation in der Bevölkerung. Kulturelle Globalisierung sorgt einerseits für weltweite Angleichungsprozesse und zeigt andererseits unterschiedliche kulturelle Perspektiven und hinterfragt den klassischen westeuropäischen Kulturkanon sowie den tendenziell engen deutschen Hochkultur-Begriff. Dies wird forciert durch eine starke Zuwanderung aus anderen Kulturkreisen, die das Kulturverständnis in der Bevölkerung insgesamt verändert (Interkulturbarometer und Keuchel 2012). Digitalisierung erweitert kulturelle Räume und Angebote, verändert die Muster sozialer Interaktion und in Folge auch die Ansprüche an Kulturrezeption im Sinne einer stärkeren Teilhabe und Mitbestimmung. Auch der Trend zur Individualisierung von Lebensstilen löst feste, milieuspezifische kulturelle Präferenzen und traditionelle Muster der Kunstrezeption auf, zugunsten individueller, selbstgestalteter Kultur-Portfolios. Dies führt zu einer Pluralisierung von Lebensweisen und kulturellen Interessen, die die Bedeutung der klassischen Kultureinrichtungen wie der Stadt- und Staatstheater schwächen könnten. „Die klassische Hochkultur hat ihre hegemoniale Kraft tendenziell verloren“ (Wimmer 2019, S. 12). Vor allem die akademischen, traditionell hochkulturaffinen Milieus haben sich zu ,kulturellen Omnivores“ (Peterson und Rossman 2007) entwickelt, in deren Lebensstil der Besuch ,hochkultureller“ Einrichtungen nur eine von vielen Aktivitäten und nicht mehr identitätsprägend ist. In einer „Gesellschaft der Singularitäten“, in der Kreativität und ästhetische Selbstinszenierung sowie -optimierung jedes Einzelnen zum Imperativ und sozialen Druck werden (Reckwitz 2012), müssen auch die klassischen Kulturanbieter für das Bedürfnis des Einzelnen nach affektgeladener Teilhabe und eigenkreativer Partizipation entsprechende Angebote vorhalten. Die rasante Entwicklung der sogenannten fünften Sparte in den öffentlichen Theatern mit Events, Workshops und partizipativen Projekten zeugt von der Reaktion auf Ansprüche nach aktiver Teilhabe und außergewöhnlichen ästhetischen Erfahrungen. Damit wird die Publikumsrolle stark aufgewertet gegenüber der traditionellen Produzentenorientierung im klassischen Kulturbetrieb in Deutschland (Mandel 2012). Die kulturpolitische Dichotomie zwischen hochkultureller Orientierung 
auf der einen und soziokultureller Teilhabeorientierung auf der anderen Seite scheint sich zunehmend aufzulösen. Im kulturpolitischen Diskurs wird bereits von der „Soziokulturalisierung des Kunst- und Kulturbetriebs“ gesprochen (Wimmer 2019; Knoblich 2018). Damit gewinne ,das Regime des Neuen auch im öffentlichen Kulturbereich die Oberhand und verdrängt die Logik des Allgemeinen, also des Kanons, des Repertoires, der Standards und der überlieferten Narrative, die bislang die traditionelle Kultur geprägt haben“. Mit Bezug auf Reckwitz spricht Sievers von der „Enthierarchisierung“ des Kulturbegriffs in der Spätmoderne (Sievers 2019, S. 24, 25).

Diese gesellschaftlichen Analysen weisen in der Konsequenz auf einen hohen Transformationsdruck für die klassischen öffentlichen Kultureinrichtungen insgesamt und die Stadt- und Staatstheater im Besonderen hin. Um die Legitimität der hohen öffentlichen Förderung zu sichern, benötigen die Stadt- und Staatstheater ausreichend Rückhalt nicht nur bei den relevanten Stakeholdern, sondern auch bei der steuerzahlenden Bevölkerung. Inwiefern repräsentiert das Theater (noch) die kulturellen Interessen in der Bevölkerung? Wer nutzt die Angebote der Theater? Welche Einstellungen zur öffentlichen Förderung von Theater und welche Erwartungen an Programm und Aufgaben von Theater gibt es in der Bevölkerung in Deutschland?

Diese Fragen sind Gegenstand einer telefonischen Repräsentativbefragung mit 1006 Interviews mit Personen aus der Grundgesamtheit der wahlberechtigten Wohnbevölkerung in Deutschland, die vom Institut für Kulturpolitik der Universität Hildesheim mit Unterstützung durch das Meinungsforschungsinstitut USUMA im Mai/Juni 2019 durchgeführt wurde. Die Befragung ist ein Bestandteil des DFG-Teilprojekts der Universität Hildesheim zu den Veränderungen der Stadtund Staatstheater durch den Strukturwandel der Publikumsnachfrage. In einem weiteren Teil geht es um die Frage, wie die Stadt- und Staatstheater diesen Strukturwandel mit veränderten kulturellen Interessen und Rezeptionsweisen im Zuge von Digitalisierung, Migration und fehlenden Enkulturationsprozessen erfahren und mit welchen Strategien sie darauf reagieren. Dies wird durch Fallstudien und Interviews an verschiedenen Theatern sowie durch eine Befragung aller Intendant*innen der Stadt- und Staatstheater erhoben. Auf welche Weise Kulturpolitik bzw. die jeweiligen öffentlichen Zuwendungsgeber Veränderungen an den Theatern proaktiv steuern, ist eine weitere Frage des Projekts.

Legitimitätsprobleme für die Stadt- und Staatstheater in Hinblick auf die Kulturnachfrage und Wertschätzung in der Bevölkerung könnten insbesondere dann entstehen: 
- wenn nur eine Minderheit der Bevölkerung Interesse an Theaterangeboten zeigt und sich das kulturelle Interesse zunehmend auf andere Kulturformen richtet;

- wenn es eine starke soziale Spaltung des Kulturpublikums gibt und Theaterangebote weitgehend nur von einer höher gebildeten und sozial eher besser gestellten Gruppe der Bevölkerung wahrgenommen werden;

- wenn Theater im Hinblick auf ihre künstlerischen und gesellschaftlichen Leistungen nicht den Erwartungen des Publikums und der Bevölkerung entsprechen und

- wenn die Förderungswürdigkeit von Stadt- und Staatstheatern von weiten Teilen der Bevölkerung infrage gestellt wird.

\section{Ergebnisse}

Die Befragung ergab dazu folgende zentrale Ergebnisse:

\subsection{Ein Drittel der Bevölkerung äußert Interesse an klassischen Kulturangeboten wie Theater - überdurchschnittlich Frauen, ältere Menschen, formal hoch Gebildete und Großstadtbewohner*innen}

Gefragt nach unterschiedlichen außerhäusigen kulturellen Aktivitäten äußerten $33 \%$ der Befragten ein Interesse an klassischen Kulturangeboten wie insbesondere Schauspiel, Oper, Klassikkonzerten oder Kunstausstellungen - im Vergleich Frauen häufiger als Männer (41\% zu $25 \%$ ); höher Gebildete häufiger als niedrig Gebildete (45\% zu $26 \%$ ); Ältere ab 60 Jahren häufiger als Jüngere zwischen 18 und 39 Jahren (40\% zu $31 \%$ ). Zu ähnlichen Größenordnungen kommt eine Befragung des Instituts für Demoskopie Allensbach (de Sombre 2017). Dabei ist zu berücksichtigen, dass das Antwortverhalten bei Fragen zu Institutionen und Sachverhalten, die von der Bevölkerung verbreitet positiv bewertet werden, von sozialer Erwünschtheit beeinflusst sein könnte.

Nischen- und Subkultur wie Jazz, Weltmusik, Kunstperformances oder Filmkunst interessiert $24 \%$ der Befragten. Ein gutes Drittel der Befragten (36\%) äußerte ein Interesse an popkulturellen Veranstaltungen wie Rock-/Popkonzerten oder populären Blockbuster-Filmen. Das stärkste Interesse erfahren mit $40 \%$ Feste und Events in der Umgebung. Während sich immerhin $71 \%$ der Bevölkerung für mindestens eine dieser Kulturformen interessieren, also im weitesten 
Sinne als „Kulturinteressierte“ gelten können, kann man $29 \%$ als „Kulturabstinente" bezeichnen, die an keiner der genannten außerhäusigen Kulturformen Interesse haben.

Generell zeigt sich: Wer sich für eine außerhäusige Kulturform interessiert, hat häufig auch ein Interesse an anderen Kulturformen.

\subsection{Nur Wenige gehören zu den „Vielbesucher*innen“ von Theatern, über die Hälfte zu den „Nichtbesucher*innen“}

Nur $10 \%$ können zu den „Vielbesucher*innen“ von Theatern generell gezählt werden (mit vier und mehr Theaterbesuchen pro Jahr), $31 \%$ zu den „Gelegenheitsbesucher*innen“ (1 bis 3 Besuche) und $59 \%$ zu den „Nichtbesucher*innen“. Der Anteil der „Vielbesucher*innen“ nimmt mit dem Bildungsniveau und dem Alter zu. Außerdem haben Frauen eine höhere Besuchsfrequenz als Männer, die ebenso wie Niedriggebildete deutlich überproportional „Nichtbesucher“ sind. Dennoch gehören vor allem auch ältere Personen mit einfacher und mittlerer Bildung zu den häufigen Besucher*innen von Theatern, wenngleich mit niedrigen Anteilen. Auffällig ist, dass auch bei den höher Gebildeten der jüngeren Generation knapp die Hälfte zu den „Nichtbesucher*innen“ gehört. Die Altersgruppe 60+mit höherer Bildung hat mit $22 \%$ den mit Abstand höchsten Anteil an „Vielbesucher*innen“.

Bildet man eine Besuchertypologie aus dem Interesse an klassischen Kulturveranstaltungen und der Besuchshäufigkeit von Theatern, so können $7 \%$ der Bevölkerung zu den „Kernbesucher*innen“ von Theatern, weitere $15 \% \mathrm{zu}$ den „,interessierten Gelegenheitsbesucher*innen“ gerechnet werden. Knapp die Hälfte der Bevölkerung (47 \%) dürften als notorische „Nie-Besucher*innen“ von Theatern eingeschätzt werden können. Die übrigen $31 \%$ gehören sonstigen Besuchertypen an. Die „Kernbesucher*innen“ setzen sich weit überproportional aus Hochgebildeten und Älteren zusammen. Männer und Niedriggebildete sind deutlich überproportional bei den „Nie-Besucher*innen“ zu finden.

Der tendenzielle Rückgang von Interesse an und Nutzung von Theatern bei der jungen Bevölkerung mit hoher Bildung, der in Längsschnittbetrachtungen durch das Institut für Demoskopie Allensbach nachgewiesen wurde (vgl. de Sombre 2017), verweist auf intergenerationelle Verschiebungen im kulturellen Geschmack und Lebensstil. Popkulturelle Veranstaltungen haben in dieser Altersgruppe eine deutlich höhere Bedeutung, und es gibt Anzeichen dafür, dass sich dies kaum verändert, wenn diese Generation älter wird. 


\subsection{Mangelnde Zeit wird noch vor mangelndem Interesse als Grund für den Nichtbesuch angegeben}

Als Hauptgrund für den Nichtbesuch oder nicht häufigeren Besuch von Stadt- und Staatstheatern wird ,mangelnde Zeit“ (36\%) am häufigsten genannt, erst dann folgt ,mangelndes Interesse“ (28\%), was ein Zeichen für die soziale Erwünschtheit von Theaterbesuchen sein könnte. Mit Abstand werden mit jeweils $12 \%$ die institutionellen Barrieren ,zu teuer“ und ,begrenzte Auswahl bzw. mangelnde Qualität“ angeführt. Nur Männer führen am häufigsten ein mangelndes Interesse an. Überdurchschnittlich häufig nennen diesen Grund auch die Jüngeren (18- bis 39-Jährige).

\subsection{Große Zustimmung zur öffentlichen Förderung von Theatern in allen Bevölkerungsgruppen, auch bei den Nichtbesucher*innen}

Die Bevölkerung stimmt weitgehend darin überein (86\%), dass die Förderung von Theatern mit Steuergeldern auch in Zukunft in bisheriger Höhe erfolgen oder sogar erhöht werden sollte. Darunter finden sich beachtliche Anteile von Personen, die nicht an Theaterangeboten interessiert sind bzw. diese selbst gar nicht wahrnehmen.

Nur $14 \%$ der Bevölkerung wollen die Förderung der öffentlichen Hand kürzen. Selbst in der Gruppe der „Nie-Besucher*innen“ sind dies nur $19 \%$. Auffällig ist, dass die jüngste Altersgruppe der 18- bis 39-Jährigen deutlich häufiger dafür plädiert, die Förderung der Theater zu kürzen als die älteren Generationen.

\section{5 Über die Produktion von Kunst hinaus sollen Stadt- und Staatstheater vor allem für eine breite Teilhabe sorgen}

Befragt nach den Erwartungen an die Theater stehen in Bezug auf die Spielplangestaltung auf den ersten Plätzen: „Programme für Kinder und Jugendliche“ (89 \%), „Programme anbieten, bei denen man lachen kann“ (86 \%) und „Stücke zeigen, die für jeden verständlich sind“ (80\%). $66 \%$ der Befragten wollen ,aktuelle Stücke und künstlerische Experimente“, $60 \%$ erwarten ,klassische Stücke von wichtigen Autor*innen“. Viele möchten sowohl klassische als auch experimentelle Stücke auf dem Spielplan sehen. Der Wunsch nach humorvollen Stücken $(86 \%)$ ist unabhängig von Bildung und Alter weit verbreitet. 
Im Hinblick auf sonstige Erwartungen an die Theater werden an erster Stelle genannt: eine „Preisgestaltung, die Menschen aus allen sozialen Schichten Teilhabe ermöglicht“ (92\%), gefolgt von der Erwartung, dass „Theater ein Treffpunkt für die breite Bevölkerung der Stadt sein sollten“ (73\%) sowie dass sie „gesellschaftliche und politische Diskussionen in der Stadt anstoßen“ (57\%). Am seltensten wird der Wunsch nach partizipativen Angeboten im Sinne von ,selber Theater spielen“ geäußert $(33 \%) .{ }^{1}$

\subsection{Die Legitimität der Stadt- und Staatstheater in der Bevölkerung scheint derzeit nicht gefährdet, es deuten sich aber mittel- und längerfristig Risiken an, die vor allem von der demografischen Entwicklung ausgehen}

Die hohe Zustimmung dafür, die Stadt- und Staatstheater auch zukünftig mindestens auf dem bisherigen Niveau mit Steuergeldern zu fördern, weit über den Kreis des Publikums hinaus, verweist auf die gesellschaftliche Bedeutung, die den Theatern zugeschrieben wird. Unabhängig vom persönlichen Nutzen scheint der über Parteigrenzen hinweg nicht infrage gestellte kulturpolitische Konsens, dass die Künste und ihre Organisationen unverzichtbar für die Gesellschaft sind, aktuell auch in der Bevölkerung noch fest verankert zu sein. Auflösungserscheinungen dieses „Rechtfertigungskonsenses“ (Schulze 2000) zeigen sich ansatzweise bei der jüngeren Generation.

Der in den Befragungsergebnissen zum Ausdruck kommende Anspruch, dass Theaterangebote für alle, unabhängig vom Einkommen, sozialen Status und Bildungsniveau, leicht zugänglich sein sollten, könnte einerseits als Indikator dafür gewertet werden, dass die Bürger*innen Theater als sozial exkludierende Einrichtung wahrnehmen, andererseits als Hinweis darauf, dass die Theater als bedeutsam für die gesamte Bevölkerung betrachtet werden.

Anhand der empirischen Befunde lässt sich als Antwort auf die oben dargestellten Hypothesen zusammenfassend konstatieren:

Nur geringe Anteile der Bevölkerung gehören zu den regelmäßigen Nutzer*innen der Theater und das Interesse in der Bevölkerung richtet sich tendenziell stärker auf unterhaltungsorientierte, popkulturelle oder nahräumliche außerhäusige Kulturaktivitäten. Auch wenn das bestehende Theaterpublikum

\footnotetext{
${ }^{1}$ Der Bericht mit den detaillierten empirischen Ergebnissen ist unter folgendem Link erhältlich: https://dx.doi.org/10.18442/077.
} 
überwiegend höher gebildet, älter und weiblich ist, stellt es keine soziodemografisch homogene Gruppe dar. Insofern kann nicht von einer klaren sozialen Spaltung gesprochen werden. Die Erwartungen an das Theater umfassen ein breites Spektrum an künstlerischen Angeboten mit sowohl klassischen wie neuen Stoffen und das Bedürfnis nach humorvollen Stücken mit hohem Unterhaltungscharakter sowie vielfältige Aktivitäten für eine breite kulturelle Teilhabe. In der Tendenz lässt sich beobachten, dass die Theater in ihrer Programmgestaltung diesen Erwartungen zu entsprechen versuchen. Allerdings ist zugleich im Theaterfachdiskurs nach wie vor eine Ablehnung unterhaltungsorientierter Stoffe zu beobachten mit Ablehnung einer „Anpassung an den Publikumsgeschmack“ (vgl. u. a. Stadttheaterdebatte auf Nachkritik.de). Eine klare Mehrheit der Bevölkerung spricht sich für die weiterhin hohe öffentliche Förderung von Theater aus, was von der Wertschätzung dieser Einrichtung zeugt.

Auch wenn angesichts dieser empirischen Befunde die Legitimation der Stadtund Staatstheater gegenwärtig offensichtlich nicht gefährdet ist, zeichnen sich dennoch Risiken für die Zukunft ab. So gibt es in den Daten dieser Befragung sowie in den Ergebnissen von Längsschnittbefragungen Hinweise auf eine mittelund längerfristig nachlassende Nachfrage nach Theaterangeboten:

Für einen bedeutenden und wachsenden Anteil auch der höher Gebildeten in den jüngeren Generationen gehören Theaterbesuche nicht mehr selbstverständlich zum Lebensstil dazu (de Sombre 2017) und es kann im Sinne einer Kohortenabhängigkeit auch nicht von einem automatischen „Hineinwachsen“ in die klassische Kultur im Alter ausgegangen werden (u. a. Reuband 2018). Vielmehr deuten sich enkulturative Brüche in Bezug auf die klassischen Kulturformen insgesamt und das Theater im Besonderen an. Die Pluralisierung kultureller Interessen und Angebote, der Wunsch nach Selbstinszenierung und aktiver Teilhabe, der sich bei den Jüngeren, die zugleich auch die „Digital Natives“ sind, auch in dieser Befragung andeutet, könnten eine nachlassende Relevanz der Theater auf dem Markt kultureller Freizeitangebote bedeuten. Allerdings zeigen die vielfältigen Aktivitäten der Stadt- und Staatstheater mit denen sie sich für neue, diverse Publikumsgruppen öffnen, Beteiligungsformate entwickeln und neue Aufgaben für die Stadtgesellschaft übernehmen, dass diese sich der Notwendigkeit bewusst sind, ihre Programme und Aufgaben an eine veränderte gesellschaftliche und demografische Situation anzupassen.

Auch wenn das Image, das die Theater selbst bei den „Nichtbesucher*innen“ genießen, derzeit nicht auf eine „Legitimationskrise“ der Theater hinweist, wirken die „Krisen-Diskurse“ der Fachöffentlichkeit offensichtlich aktivierend auf die Theaterschaffenden. Entsprechend einer Interpretation der Kunstfreiheitsgarantie 
als Freiheit der öffentlichen Kunstinstitutionen vor staatlichen Interventionen, halten sich die Träger der Theater zurück mit Vorgaben für Ziele und Maßnahmen, solange die Auslastungszahlen einigermaßen auf eine Akzeptanz beim Publikum hinwiesen, so die übereinstimmende Einschätzung in einer qualitativen Befragung von Leitungen öffentlicher Theater (Mandel 2018 sowie Mandel 2020 in diesem Band). Kulturpolitik begegnet den öffentlichen Theatern eher diffus und wenig transparent, etwa auch in der Besetzungspolitik von Intendanzen. Mit welchen Maßnahmen sich die Theater an veränderte gesellschaftliche Bedingungen anpassen, bleibt ihnen offensichtlich weitgehend selbst überlassen. In einer solchen Situation liegt es den Annahmen der neo-institutionalistischen Organisationstheorie nach nahe, sich dabei an anderen, als erfolgreich geltenden Einrichtungen ihres Referenzsystems zu orientieren (Walgenbach 2006; von Cossel 2011). So erklärt sich, dass viele Theater ähnliche neue Ansätze entwickeln, etwa im Bereich partizipativer Theaterarbeit, mit denen sie gesellschaftliche Relevanz zeigen wollen. Um Legitimität gegenüber ihren Trägern und anderen wichtigen Anspruchsgruppen einschließlich ihres Stammpublikums zu sichern, müssen die Theater zugleich den Kriterien einer zufriedenstellenden Auslastung bei einem vielfältigen Spielplan Genüge tun, sich der Nachfrage und den Bedürfnissen ihres aktuellen Publikums anpassen und gleichzeitig neue Strategien entwickeln, um darüber hinaus ganz andere Zielgruppen zu erreichen. Widersprüchlichen Erwartungen versuchen Theater vor allem mit der Strategie der „Entkoppelung“ zu begegnen, indem sie neue Aktivitäten als „Add-on“ in ihr Portfolio integrieren (z. B. „fünfte Sparte“) ohne sich von alten Handlungsmustern und Wertsystemen lösen zu müssen (Mandel 2013). Dabei werden Überforderungstendenzen deutlich, wenn die Theater unter weitgehender Beibehaltung ihrer traditionellen Arbeitsweisen immer mehr Aufgaben übernehmen und neue Formate entwickeln. Die langjährig gewachsenen organisatorischen Strukturen, die Sparten, Gewerke, Abteilungen und damit verbundene Hierarchien sowie eine gefestigte Organisationskultur aus Handlungsroutinen und unter Mitarbeiter*innen breit geteilten Werthaltungen und Glaubenssätzen können Transformationsprozesse erschweren (Mandel 2018). Eine proaktive Unterstützung durch klare kulturpolitische Ziele für veränderte Prioritäten der Theater in der "Stadtgesellschaft“ könnte die notwendigen Transformationsprozesse in den Strukturen der Einrichtungen unterstützen. 


\section{Literatur}

de Sombre, Steffen. 2017. AWA - Bildungsbürgertum und Massenkultur. https://de.slides hare.net/hemartin/awa-2017-dr-steffen-de-sombre-bildungsbrgertum-und-massenkultur. Zugegriffen: 07. Jan. 2020.

Interkulturbarometer, und Keuchel, Susanne. 2012. Das 1. InterKulturBarometer. Migration als Einflussfaktor auf Kunst und Kultur. Zentrum für Kulturforschung. Köln: Arcult.

Knoblich, Tobias. 2017. Programmformeln und Praxisformen von Soziokultur - Kulturpolitik als Kulturelle Demokratie. Wiesbaden: Springer VS.

Mandel, Birgit. 2012. Audience Development als Aufgabe von Kulturmanagementforschung. In Jahrbuch für Kulturmanagement, 15-27. Im Auftrag des Fachverbandes für Kulturmanagement. Bielefeld.

Mandel, Birgit. 2013. Interkulturelles Audience Development. Zukunftsstrategien für öffentlich geförderte Kultureinrichtungen. Bielefeld: transcript.

Mandel, Birgit. 2018. Veränderungen im Cultural Leadership durch neue Generationen von Führungskräften? Ergebnisse einer Befragung von älteren und jüngeren Führungskräften in öffentlichen Kultureinrichtungen in Deutschland. Hildesheim: Universitätsverlag. https://doi.org/10.18442/823.

Mandel, Birgit. 2020. Das (un-)entbehrliche Theater? Die Bedeutung der Stadt- und Staatstheater für die ,Stadtgesellschaft“. Einführung in das Teilkapitel. In diesem Band.

Peterson, R., und G. Rossman. 2007. Changing arts audiences: Capitalizing on Omnivorousnes. In Engaging art: The next great transformation of American's cultural life, Hrsg. B. Ivey und S. Tepper, 307-342. New York: Routledge.

Reckwitz, Andreas. 2017. Die Gesellschaft der Singularitäten. Zum Strukturwandel der Moderne. Berlin: Suhrkamp.

Reckwitz, Andreas. 2012. Die Erfindung der Kreativität. Berlin: Suhrkamp.

Reuband, Karl-Heinz. 2018. Kulturelle Partizipation in Deutschland. In Jahrbuch für Kulturpolitik 2017/18, Hrsg. U. Blumenreich, S. Dengel, W. Hippe, N. Sievers, 377-393. Institut für Kulturpolitik der Kulturpolitischen Gesellschaft. Bielefeld: transcript Verlag.

Schulze, Gerhard. 2000. Die Erlebnisgesellschaft: Kultursoziologie der Gegenwart. Frankfurt a.M.: Campus.

Sievers, Norbert. 2019. Erklärungen, Methoden und Praxisformen soziokultureller Projektarbeit. In Neue Methoden und Formate der soziokulturellen Projektarbeit, Hrsg. U. Blumenreich, F. Kröger, L. Pfeiffer, N. Sievers und C. Wingert, 21-27. Bonn: Institut für Kulturpolitik der Kulturpolitischen Gesellschaft.

Von Cossel, Friederike. 2011. Entscheidungsfindung im Kulturbetrieb am Beispiel der Spielplangestaltung im Theater. Augsburg: Hampp.

Walgenbach, Peter. 2006. Neoinstitutionalistische Ansätze in der Organisationstheorie. In Organisationstheorien, Hrsg. A. Kieser und M. Ebers, 353-401. Stuttgart: Kohlhammer.

Wimmer, Michael. 2019. Soziokultur im gesellschaftlichen und kulturpolitischen Wandel. In Neue Methoden und Formate soziokultureller Projektarbeit, Hrsg. U. Blumenreich, F. Kröger, L. Pfeiffer, N. Sievers und C. Wingert, 28-35. Bonn: Institut für Kulturpolitik der Kulturpolitischen Gesellschaft. 
Open Access Dieses Kapitel wird unter der Creative Commons Namensnennung 4.0 International Lizenz (http://creativecommons.org/licenses/by/4.0/deed.de) veröffentlicht, welche die Nutzung, Vervielfältigung, Bearbeitung, Verbreitung und Wiedergabe in jeglichem Medium und Format erlaubt, sofern Sie den/die ursprünglichen Autor(en) und die Quelle ordnungsgemäß nennen, einen Link zur Creative Commons Lizenz beifügen und angeben, ob Änderungen vorgenommen wurden.

Die in diesem Kapitel enthaltenen Bilder und sonstiges Drittmaterial unterliegen ebenfalls der genannten Creative Commons Lizenz, sofern sich aus der Abbildungslegende nichts anderes ergibt. Sofern das betreffende Material nicht unter der genannten Creative Commons Lizenz steht und die betreffende Handlung nicht nach gesetzlichen Vorschriften erlaubt ist, ist für die oben aufgeführten Weiterverwendungen des Materials die Einwilligung des jeweiligen Rechteinhabers einzuholen.

(c) (1) 